\title{
Study of Fitting Models on Moisture Sorption Isotherms of Profiled Polyester Fabric
}

\author{
Hong-ru Liu ${ }^{1, a^{*}}$, Shang-ping $\mathrm{Wu}^{2, b}$ \\ 1 School of Materials Science and Engineering, Beijing Institute of Fashion Technology, Beijing \\ 100029, China \\ 2 Datang International Power Generation Co of Beijing High Well Thermal Power Plant, Beijing \\ 100041,China \\ a lucylhr@163.com,b wushangping@sina.com
}

\begin{abstract}
Keywords: profiled polyester, moisture sorption isotherms, equilibrium moisture content
Abstract: In this paper, the moisture sorption isotherms were measured of the profiled polyester (trefoil-shaped) fabric at different temperature and relative humidity, the influence of the temperature, relative humidity were summarized, and three kinds of commonly were used empirical models of moisture sorption comparing the fitting of experimental data, the suitable profiled polyester empirical model of equilibrium moisture content of fabric were identified. The results showed that with the increase of temperature, weave the equilibrium moisture content rate decreased slightly; with the increase of relative humidity, weave the equilibrium moisture content rate increased, and relative humidity than the temperature has greater effect; the model can agree well with the experimental data.
\end{abstract}

\section{Introduction}

Fabric wet comfort performance has been the focus of industry research, moisture adsorption capacity of the fiber is easy to absorb human body discharges sweat, thermoregulation, relieve stuffy wet sense, which makes people feel comfortable. Because of the non hydrophilic groups, the fiber has the poor moisture absorption ability, which makes it much worse in comfort than the cotton and other natural fibers.. For profiled polyester fiber, due to the presence of surface grooves and micro porous, the formation of capillary number increases, the fabric has greater improvement in the breathable, absorbent, breathable and so on. ${ }^{[1-3]}$

Moisture sorption isotherm is an important tool for studying the relationship between the adsorption substrate and moisture. At present, the study on the moisture adsorption isotherms of the fabric is very little at home and abroad. ${ }^{[4-6]}$ In this paper trefoil PET fabric moisture absorption isotherms were measured under different temperature and humidity conditions, and the experimental data were fitted using the commonly used three kinds of empirical model, suitable for profiled polyester fabric flat

\section{Materials and Instruments}

\section{Experimental Materials}

Test samples for the profiled polyester ((trefoil-shaped) fabric, warp and weft density of $480 \times 300$ (root $/ 10 \mathrm{~cm}$ ), the thickness $0.42 \mathrm{~mm}$, gram weight $116.5 \mathrm{~g} / \mathrm{m}^{2}$.

\section{Experimental Apparatus}

SDH0501 type low temperature humid heat test box, Chongqing Yinhe experimental instruments; EX324 electronic analytical balance, Ohaus instruments (Shanghai) Co., Ltd., oven, Ningbo Textile Instrument Factory. 


\section{Experimental Method}

\section{Determination and Calculation of Moisture Content of Moisture Absorption Equilibrium}

Moisture absorption and moisture content of moisture content is the maximum moisture content of fabric under certain environmental conditions (certain temperature and relative humidity).. At the constant temperature, the relationship curve between the moisture absorption equilibrium moisture content (dry basis) and relative humidity RH is called the moisture sorption isotherm.

This experiment according to GB / T9995.1997 "textile materials moisture content and moisture regain determination of an oven drying method, standard and in low temperature and moist heat test box of tissue hygroscopic material balance experiment. To ensure the experimental conditions close to the actual human environment, the temperature is set at $35,40,65 \%, 70 \%, 80 \%, 75 \%, 85 \%, 90 \%$, $95 \%, 45$.

That take in the oven drying 2.5 hours after the fabric dry weight, and fabric object is arranged on the set environment, at regular intervals on the fabric were weighed until fabric of constant weight, that reach equilibrium moisture.

The formula for calculating the equilibrium moisture content (dry basis) is:

$\mathrm{Me}=\left[\left(\mathrm{W}-\mathrm{W}_{0}\right) / \mathrm{W}_{0}\right] \times 100 \%(1)$

$\mathrm{W}$ - the weight of the fabric in the condition of certain environment can reach the equilibrium of moisture absorption.

$\mathrm{W}_{0}$ - dry weight of the fabric

\section{Moisture Content Model of Moisture Absorption Equilibrium}

Many scholars in the world have carried out a lot of research on the adsorption theory, and have proposed many adsorption theory and experience model, but these models can be established only in a certain moisture content and temperature range.. So far, no theoretical model can accurately describe the equilibrium moisture content of the material.. At present, only a few semi theoretical empirical models are used to describe the equilibrium moisture content of materials, which are widely used in the application of the theory.In this paper, 3 commonly used empirical models are used to fit the experimental data, and the model is shown in Table 1.

Table 1 Three models of equilibrium moisture content

\begin{tabular}{ccc}
\hline Model & Equations of equilibrium & Adsorption theory basis \\
\hline Modify-Oswin & $\begin{array}{c}\mathrm{Me}=(\mathrm{A}+\mathrm{BT})[\mathrm{RH} /(1-\mathrm{RH}) \\
]^{\mathrm{C}}\end{array}$ & $\begin{array}{r}\text { Fitting results of a large } \\
\text { number of experimental data }\end{array}$ \\
Modify-Henderson & $\mathrm{Me}=\{-\ln (1-\mathrm{RH}) /[\mathrm{A}(\mathrm{B}+\mathrm{T})$ & Gibbs adsorption theory \\
Modify-Chung-Pfo & ]$^{\mathrm{C}}$ & $\begin{array}{c}\mathrm{Me}=\mathrm{C} \ln \{\mathrm{A} /[(\mathrm{T}+\mathrm{B}) \\
\text { st }\end{array}$ \\
& $(\operatorname{lnRH})]\}$ & Polanyi adsorption \\
\end{tabular}

Note: in the model, $\mathrm{RH}$ is relative humidity (fractional), Me is the material equilibrium moisture content ( $\%$, dry basis), $\mathrm{T}$ is the ambient temperature (c), B, C, A are constant, and the material properties are related. 


\section{Results and Discussion}

\section{Moisture sorption isotherms of profiled polyester fabrics}

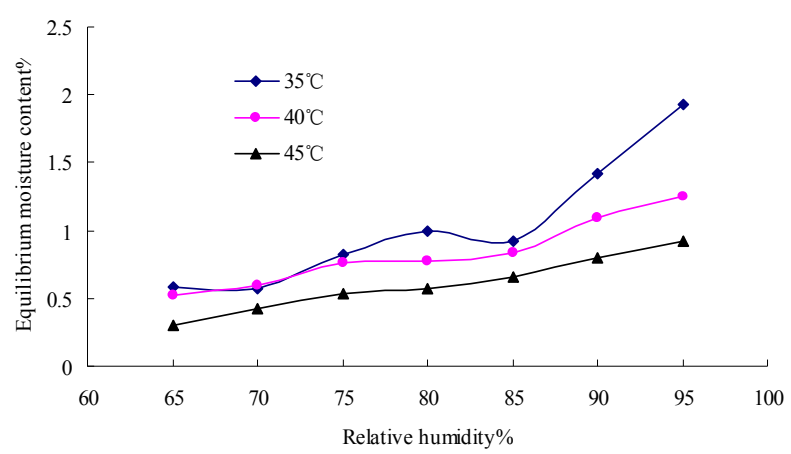

Fig. 1 Moisture sorption isotherms of profiled polyester fabric at different temperatures

Adsorption isotherms of the profiled polyester fabric at different temperatures were shown in Fig. 1. Can be seen from the picture, the equilibrium moisture content decreases slightly with the increase of temperature.. With the increase of relative humidity, equilibrium moisture content increases, the different range of relative humidity, equilibrium moisture content changes in different, when the relative humidity is greater than $85 \%$, the moisture balance rate increased rapidly, and the increasing values significantly greater than the increased with increasing temperature and decreasing value of the resulting. It is fully explained that the relative humidity ratio has a greater impact on the equilibrium moisture content.

\section{Model Simulation}

Table 2 Simulation results of moisture isothermal model of the profiled polyester fabric

\begin{tabular}{ccccc}
\hline Model & $\mathrm{A}$ & $\mathrm{B}$ & $\mathrm{C}$ & $\mathrm{R}^{2}$ \\
\hline Modify-Oswin & 1.3012 & -0.02258 & 0.4317 & 94.13 \\
Modify-Henderson & 0.1238 & -21.6613 & 1.018 & 99.53 \\
Modify-Chung-Pfost & -42.0317 & -9.1509 & 0.4187 & 93.87
\end{tabular}

The model constants and fitting effect of the model were obtained by using Mathematical Wolfram software,the results were shown in Table 2. From the Table 2, the Modify-Henderson model is used to fitting the moisture content of the moisture content of the shaped polyester fabric with the moisture content of the wet polyester fabric, and the moisture balance equation of the shaped PET fabric is determined.

$$
\mathrm{Me}=\{-\ln (1-\mathrm{RH}) /[0.1238(\mathrm{~T}-21.6613)]\}^{1.018}
$$

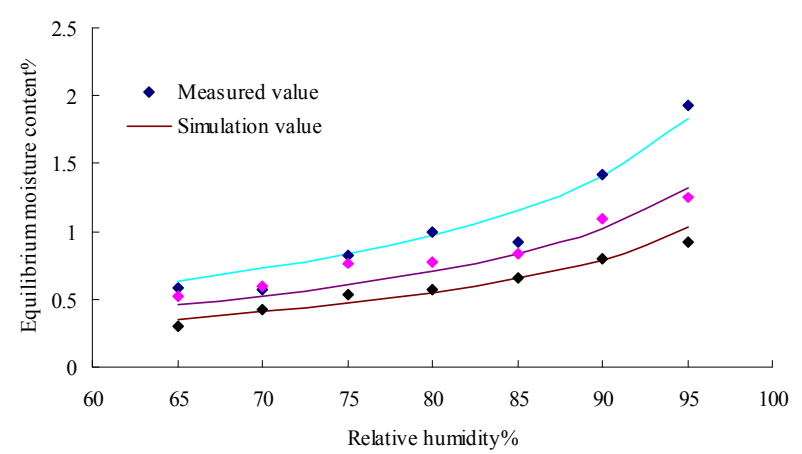

Fig. 2 The relationship between the measured value of the fabric of the profiled polyester 
the relationship between the measured value of the fabric of the profiled polyester fabric and the model of the Modify-Henderson model were shown in Fig. 2. It can be seen that the simulated values are in good agreement with the experimental values, and the change of the two trends is the same, and the moisture sorption isotherm curves changes. Thus it may be known, the model can a woven fabrics under given conditions of isothermal absorption curve simulation of very good, can be used to predict under certain temperature and relative humidity on equilibrium moisture content and for other fabric hygroscopic material model was established to provide a reference.

\section{Conclusions}

(1) The relative humidity and temperature have some effect on the equilibrium moisture content of the profiled polyester, and the relative humidity has a greater effect than the temperature.

(2) Modify-Henderson model can predict the equilibrium moisture content of the profiled polyester fabric under certain environmental conditions..

\section{Acknowledgements}

Fund Project: The Beijing Municipal Board of Education Projects(KM201510012007)

\section{References}

[1] Yueling Liu,Profiled modified polyester fabric with cotton fabric properties contrast [J]. Cotton Textile Technology, 39, 2011 (8): 26-28.

[2] Fang He, Jingyi Zhou, Plain fabric wet comfort properties of [J]. Journal of Tianjin University of Technology, 2011, 30 (1): 32-35

[3] Lingjie Liu, Yingchun Du. Simulating moisture absorption of fabric through molecular layer [J] Journal of Textile Research, 2008,29 (7): 30-33

[4] Brojeswari Das et al. Studies on moisture transmission properties of PV-blended fabrics [J], The Journal of The Textile Institute.October 2009,Vol.100, No.7,588-597.

[5] Cui-fang Li et al.Study on the moisture absorption performance of Profiled polyester fabric[C],The 12th Asian Textile Conference. 2013.11:1282-1287

[6] Hong-ru Liu et al.Study on Moisture Transfer Properties of Polyester-Cotton Fabric[C], The 2013 International Conference on Applied Science and Engineering Technology ,2013.4,Vol.709 : 211-214 